\title{
An effective strategy to prevent allopurinol-induced hypersensitivity by HLA typing
}

\author{
Jae-Woo Jung, MD, PhD 1,2, Dong-Ki Kim, MD, PhD³, Heung-Woo Park, MD, PhD 1,3, \\ Kook-Hwan Oh, MD, PhD³, Kwon-Wook Joo, MD, PhD³, Yon-Su Kim, MD, PhD, Curie Ahn, MD, PhD³, \\ Kyung Wha Lee, $\mathrm{PhD}^{4}$, Sang-Heon Cho, MD, PhD ${ }^{1,3}$, Kyung-Up Min, MD, $\mathrm{PhD}^{1,3}$ \\ and Hye-Ryun Kang, MD, PhD ${ }^{1,3}$
}

\begin{abstract}
Purpose: This study was conducted to evaluate the usefulness of human leukocyte antigen (HLA) typing in preventing allopurinolinduced severe cutaneous adverse reactions (SCARs) through the application of an allopurinol tolerance induction protocol or prescription of other alternative medications in high-risk patients.
\end{abstract}

Methods: HLA typing was performed in patients with chronic renal insufficiency who needed allopurinol. HLA-B ${ }^{\star}$ 58:01-negative patients were prescribed the usual dose of allopurinol. For HLA$B^{\star} 58: 01$-positive patients, administration of either allopurinol based on a 28 -day tolerance induction protocol or alternative medications was initiated. Hypersensitivity reactions were surveyed for 90 days and compared with the result of a previous retrospective cohort study.

Results: Among a total of 401 study subjects, no SCARs were noted in HLA-B ${ }^{\star} 58: 01$-positive patients with application of the tolerance induction protocol $(n=30)$ or alternative medications $(n=16)$, nor were any SCARs observed in HLA-B ${ }^{\star} 58$ :01-negative patients who started allopurinol at the usual dose $(n=355)$. Compared with the previous retrospective cohort study, a significant reduction in SCARs was observed in HLA-B ${ }^{\star} 58: 01$-positive patients ( 0 vs. $18 \% ; P=0.002$ ).

Conclusion: This study shows the usefulness of HLA-B ${ }^{\star} 58: 01$ screening in identifying patients at high risk for the development of allopurinol-induced SCARs and suggests that application of a tolerance induction protocol or alternative medications could be an effective strategy to prevent allopurinol-induced SCARs in HLA$B \star 58: 01-$ positive patients.

Genet Med advance online publication 29 January 2015

Key Words: allopurinol; drug hypersensitivity; HLA-B58:01 antigen; immune tolerance; renal insufficiency, chronic

of SCARs when they prescribe allopurinol. ${ }^{8}$ Chronic renal insufficiency (CRI) is a well-known risk factor for allopurinolinduced SCARs and is an underlying disease that needs an allopurinol prescription for the related hyperuricemia.

The association between the occurrence of severe drug hypersensitivity reactions and human leukocyte antigen (HLA) alleles has recently been reported. In Han Chinese patients, 72 $100 \%$ of those with carbamazepine-induced SJS/TEN had HLA$B^{\star} 15: 02$ (refs. 9,10). In Caucasian patients, $44-94 \%$ of those with abacavir hypersensitivity were HLA-B ${ }^{\star 57: 01-\text { positive. }^{11,12}}$ Patients with allopurinol-induced SCARs also showed a strong association with HLA-B ${ }^{\star 58: 01}$ in studies performed in Asians, such as Han Chinese, Thais, Vietnamese, and Koreans. In particular, 100\% of Han Chinese and Thai patients with allopurinol-induced SCARs showed positivity for HLA-B ${ }^{\star} 58: 01 .{ }^{6,13,14}$

In our previous case-control study we observed a very strong positive association between allopurinol-induced SCARs and the HLA-B ${ }^{\star} 58: 01$ allele in a Korean population (odds ratio $=$ 97.8; 95\% confidence interval: $18.3-521.5) .{ }^{14}$ In our retrospective cohort study reported in 2011, a strong positive association between allopurinol-induced SCARs and HLA-B ${ }^{\star} 58: 01$ was

\footnotetext{
${ }^{1}$ Institute of Allergy and Clinical Immunology, Seoul National University Medical Research Center, Seoul, Korea; ${ }^{2}$ Department of Internal Medicine, Chung-Ang University College of Medicine, Seoul, Korea; ${ }^{3}$ Department of Internal Medicine, Seoul National University College of Medicine, Seoul, Korea; ${ }^{4}$ Hallym Institute for Genome Application, Hallym University College of Medicine, Anyang, Korea. Correspondence: Hye-Ryun Kang (helenmed@snu.ac.kr)
} 


\section{ORIGINAL RESEARCH ARTICLE}

confirmed in Korean patients with CRI (odds ratio $=179.24$; 95\% confidence interval: 10.19-3,151.74 (ref. 15). These associations between SCAR development and certain HLA alleles provide us with a pharmacologic screening tool to avoid the serious morbidity and mortality of SCARs in high-risk patients who possess a certain HLA allele.

For patients who are hypersensitive to a certain drug, reintroduction of an etiologic agent should be prohibited, and choosing alternative drugs is recommended. To determine whether to continue using the culprit drug, a tolerance induction procedure-a procedure to induce immune tolerance based on a desensitization protocol-has recently been increasingly used in patients with a drug allergy. ${ }^{16}$

We performed this study to evaluate the clinical usefulness of (i) HLA-B ${ }^{\star} 58: 01$ screening and (ii) application of an allopurinol tolerance induction protocol or administration of alternative medications for the primary prevention of allopurinol-induced SCARs in high-risk patients by comparing our results with the incidence of allopurinol hypersensitivity of a previous retrospective cohort study of a similar population.

\section{Study subjects}

\section{MATERIALS AND METHODS}

This study was performed from March 2011 to November 2012 with the permission of the institutional review board of Seoul National University Hospital (H-1009-009-331). The study subjects were allopurinol-naive patients with CRI older than 18 years of age who needed allopurinol medication for the treatment of gout or hyperuricemia. For comparative analysis of clinical characteristics, the following data were collected: each patient's age, sex, height, body weight, white blood cell count with differentials, liver function, renal function, and uric acid concentration. Estimated creatinine clearance was calculated using the Cockcroft-Gault formula. History of underlying disease and concomitant administration of thiazide, another risk factor for allopurinol-induced SCARs or immunomodulatory drugs, were also assessed.

\section{HLA typing}

Genomic DNA was extracted from white blood cells in peripheral blood using the salting out method. ${ }^{17} H L A-B$ alleles were analyzed by direct DNA sequence analysis with well-established protocols. ${ }^{18,19}$

\section{Study flows}

HLA-B tests were done after receiving the written consent of the patients (Figure 1). If the patients were HLA-B ${ }^{\star} 58$ :01-negative, allopurinol was started at 50 or $100 \mathrm{mg}$, according to their creatinine clearance. If patients were HLA-B`58:01-positive and agreed to allopurinol administration, allopurinol was started at $50 \mu \mathrm{g} / \mathrm{day}$ on the first day and slowly escalated to $100 \mathrm{mg} /$ day on 28th day, according to the tolerance induction protocol. Subsequently, dose was adjusted based on the renal clearance of individual subjects in either the HLA-B ${ }^{\star 58: 01-p o s i t i v e ~ o r ~}$
JUNG et al | Screening test for HLA-B*58:01 and prevention of allopurinol-induced SCARs

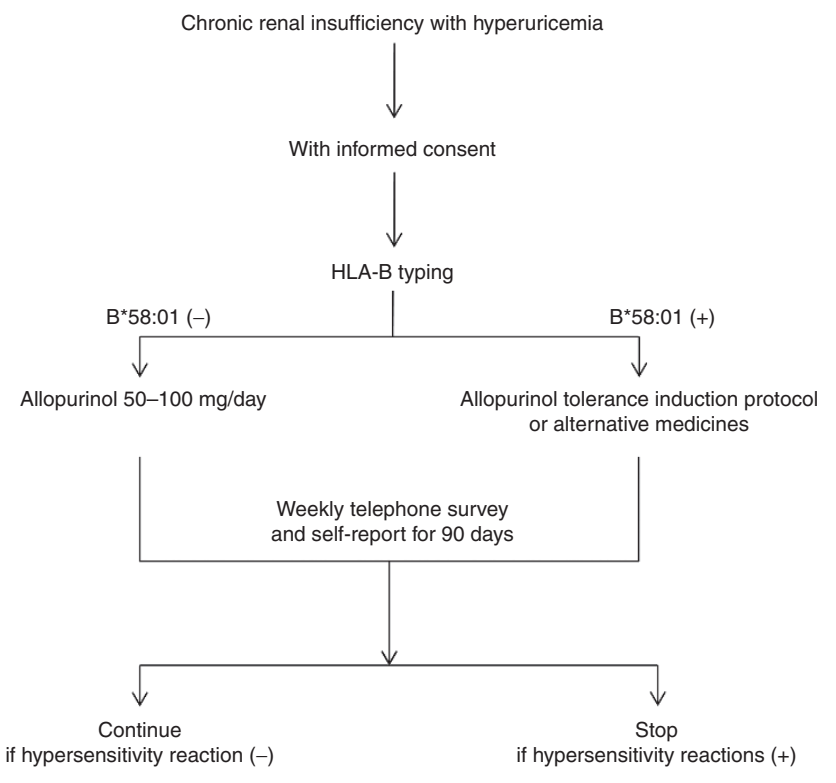

Figure 1 Prospective study to prevent allopurinol-induced severe cutaneous adverse reactions (SCARs) in high-risk patients. HLA, human leukocyte antigen.

-negative group. ${ }^{20}$ Because the target serum uric acid concentration for the treatment of hyperuricemia is still controversial in chronic kidney disease, it was determined by nephrologists; the recommended target concentration was less than $6 \mathrm{mg} / \mathrm{dl}$ for the treatment of gout.

When allopurinol hypersensitivity reactions were diagnosed, allopurinol administration was stopped immediately. If there were suspicious symptoms suggesting hypersensitivity reactions between telephone surveys, the patients were educated to report their symptoms to an investigator immediately. When patients withdrew their informed consent or discontinued allopurinol because of adverse reactions other than hypersensitivity or improvement of hyperuricemia before the end of the 90-day study period, they were not included in the analysis.

If patients were HLA-B ${ }^{\star} 58: 01$-positive and did not agree to allopurinol administration, benzbromarone or febuxostat was administered. The patients were followed up weekly for 90 days by telephone survey about the presence of a hypersensitivity reaction. To see the effectiveness of HLA-B ${ }^{\star} 58: 01$ screening itself, HLA- $\mathrm{B}^{\star} 58: 01$-positive patients treated with alternative medicines were grouped together with HLA- $B{ }^{\star} 58$ :01-positive patients who underwent allopurinol tolerance induction.

Based on the study flow mentioned above, the incidence of allopurinol-induced SCARs based on the absence or presence of the HLA-B ${ }^{\star}$ 58:01 allele was assessed. In addition, we compared the incidence of SCARs induced by allopurinol before and after introduction of HLA-B screening to prove the usefulness of the tolerance induction protocol for the prevention of SCARs in high-risk patients. The incidence assessed in our retrospective cohort study was used as a historical control. ${ }^{11}$ 


\section{Allopurinol tolerance induction protocol}

Patients who were HLA-B ${ }^{\star 58: 01-p o s i t i v e ~ b a s e d ~ o n ~ t h e ~}$ HLA screening test were prescribed allopurinol following a 28-day schedule for the slow oral tolerance induction protocol $^{21}$ (Supplementary Table S1 online). The allopurinol tolerance induction protocol used in this study was originally made for patients who had allopurinol-induced maculopapular eruptions. It is a slow tolerance induction protocol that starts with a dose of $50 \mu \mathrm{g}$ allopurinol and is doubled at 3 -day intervals, reaching $100 \mathrm{mg}$ on the 28th day. After the 28 th day, the allopurinol dose was titrated, taking into consideration the serum creatinine and uric acid concentrations, from day 29 to day 90.

\section{Definition of hypersensitivity reaction to allopurinol}

Allopurinol hypersensitivity reaction was defined as cutaneous symptoms such as pruritus, urticaria, maculopapular eruption, and erythema that develop after the introduction of allopurinol and then disappear with the discontinuation of allopurinol. SCARs include SJS, TEN, and allopurinol hypersensitivity syndrome. ${ }^{8,22}$ SJS was defined as skin detachment across less than $10 \%$ of the body surface area, SJS/TEN was defined as detachment between 10 and $30 \%$ of the body surface area, and TEN was defined as detachment of more than $30 \%$ of the body surface area. ${ }^{22}$ Allopurinol hypersensitivity syndrome was defined as present when patients with a clear history of exposure to allopurinol met at least two major criteria, including worsening of renal function, acute hepatocellular injury, and a generalized rash, or one of the major criteria plus at least one of the following minor criteria: fever, eosinophilia, and leukocytosis. ${ }^{8}$

Allopurinol hypersensitivity reactions were evaluated by two board-certified allergy specialists and defined as a case of certain or probable causality based on the World Health Organization Adverse Reaction Terminology assessment. ${ }^{23}$

\section{Statistical analysis}

The sample size was determined taking into account a 20\% dropout rate and a significant reduction of SCARs in the HLA$B \star 58: 01$ group by avoiding allopurinol (usual dosage) with HLA-B screening. To see the significant reduction in the incidence of SCARs to $4 \%$ or less from the previous $18 \%$, at least 33 HLA-B ${ }^{\star}$ 58:01-positive patients with CRI were needed when using PASS 2008 software (NCSS, Kaysville, UT). To get 33 subjects with HLA-B*58:01, a total of 345 Korean subjects were required. Considering that the phenotype frequency of HLA$B^{\star}$ 58:01 in South Korea is $12.2 \%$ (ref. 19) and the previous incidence rate of $18 \%$ in HLA-B ${ }^{\star 58: 01-p o s i t i v e ~ p a t i e n t s ~ w i t h ~ C R I, ~}{ }^{15}$ the estimated number of patients prone to allopurinol-induced SCARs was 7.58 of 345 Korean subjects with CRI.

Statistical analysis was performed with SPSS version 16.0 (SPSS, Chicago, IL). Continuous variables were expressed as means and SD; categorical variables were expressed as a number and percentage. To compare the clinical characteristics according to the presence of HLA-B ${ }^{\star} 58: 01$, the $\chi^{2}$ test, twotailed Fisher's exact test, and Mann-Whitney test were used.
To test the preventive effect of HLA-B ${ }^{\star} 58$ :01 screening for serious allopurinol hypersensitivity, we compared the incidence of allopurinol hypersensitivity in the current prospective study to that of our previous retrospective cohort study, which had been carried out using a similar study population in the same institution. $P<0.05$ was considered statistically significant.

\section{RESULTS}

\section{Characteristics of the study population}

A total of 425 patients were screened for HLA-B ${ }^{\star}$ 58:01. Fortyeight patients $(11.3 \%)$ were HLA-B ${ }^{\star} 58: 01$-positive and 377 patients $(88.7 \%)$ were HLA-B ${ }^{\star} 58: 01-$ negative. Two of the 48 HLA-B ${ }^{\star 58: 01-p o s i t i v e ~ p a t i e n t s ~ a n d ~} 6$ of the 377 HLA-B ${ }^{\star 58: 01-~}$ negative patients withdrew their consent. In addition, 16 HLA-B ${ }^{\star}$ 58:01-negative patients were excluded from the analysis because of protocol violation; other reasons for exclusion included incompliance in 8 patients, discontinuation of allopurinol due to adverse reactions other than hypersensitivity in 4 patients, and no need for allopurinol maintenance due to rapid improvement of the urate concentration in 4 patients. Therefore, a total of 401 patients, 46 HLA-B ${ }^{\star 58: 01-p o s i t i v e ~}$ and 355 HLA-B ${ }^{\star 58: 01-n e g a t i v e, ~ w e r e ~ s u b j e c t e d ~ t o ~ t h e ~ a n a l y s i s ~}$ (Figure 2).

The mean age of the cohort was $58.15 \pm 15.49$ years, and it included 287 men (71.6\%). There were no differences in age, sex, body mass index, liver function, renal function, or pre- as well as post-allopurinol uric acid concentrations between the HLA-B ${ }^{\star} 58$ :01-positive and the HLA-B ${ }^{\star} 58$ :01-negative groups (Table 1). The final allopurinol dose was not different between these groups $(103.45 \pm 42.11$ vs. $95.00 \pm 29.26 \mathrm{mg} ; P=0.151)$. In addition, history of coadministration of thiazide or immunosuppressive drugs, such as systemic steroids and cyclosporine, and underlying diseases including allergic disease, did not differ between groups.

\section{Allopurinol hypersensitivity according to the presence of HLA-B*58:01}

Allopurinol was administrated via a tolerance induction protocol $(n=30)$ or substituted with benzbromarone $(n=11)$ or febuxostat $(n=5)$ in 46 patients with the HLA-B ${ }^{\star}$ 58:01 allele. During the 90-day period of drug administration, none of the 46 patients with the HLA-B ${ }^{\star 58: 01}$ allele developed SCARs. Only one patient $(2.2 \%)$ experienced a very mild rash. This 75-year-old male patient had only several erythematous papules without fever or mucosal involvement on the 73rd day, and the skin lesions rapidly improved 4 days after ceasing the administration of allopurinol.

Although none of the 355 HLA-B ${ }^{\star} 58$ :01-negative patients taking the usual dose of allopurinol experienced SCARs, 17 patients (4.8\%) developed simple maculopapular eruptions (Figure 2). Their mean age was $63.94 \pm 12.34$ years, and 12 (70.6\%) were men. The mean dose of allopurinol was $76.47 \pm 25.72 \mathrm{mg}$, and the onset of rash occurred $35.18 \pm 24.70$ days after starting the allopurinol medication. Maculopapular eruption disappeared after discontinuation of allopurinol. There were no significant 


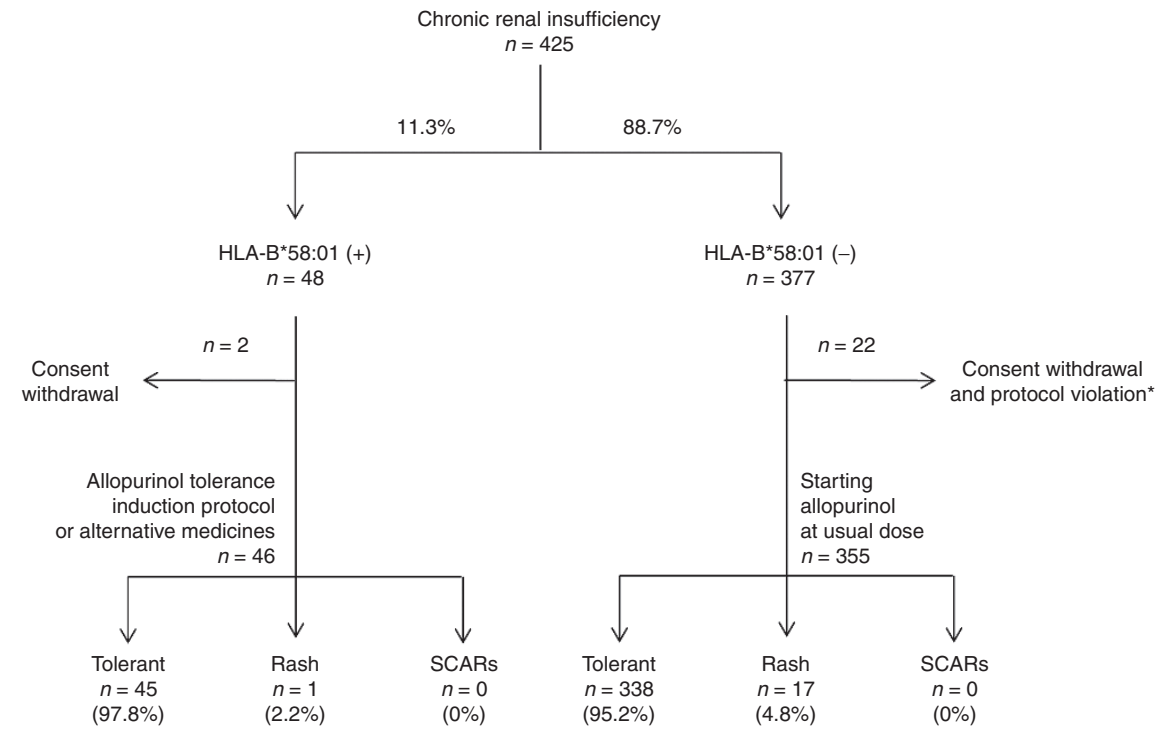

Figure 2 Flowchart of study design. Among a total of 425 patients with chronic renal insufficiency, 401 patients completed the study, and none of them experienced severe cutaneous adverse reactions (SCARs). *Withdrawal of allopurinol regardless of hypersensitivity.

Table 1 Comparison of clinical characteristics according to the presence of HLA-B*58:01

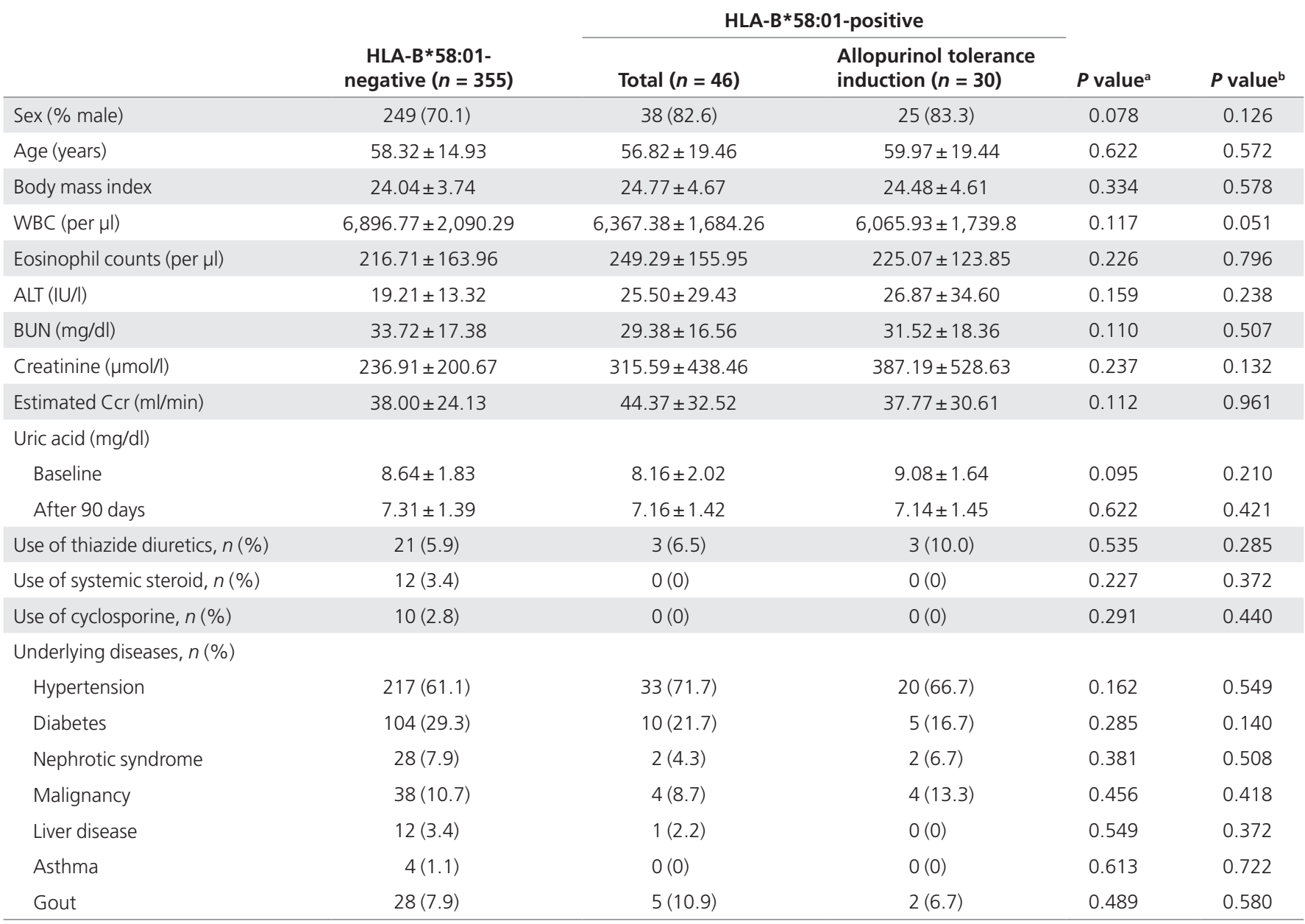

ALT, alanine aminotransferase; BUN, blood urea nitrogen; Ccr, creatinine clearance; HLA, human leukocyte antigen; WBC, white blood cell count.

${ }^{a}$ Comparison between HLA-B*58:01-negative patients and HLA-B*58:01-positive patients. ${ }^{b}$ Comparison between HLA-B*58:01-negative patients and HLA-B*58:01-positive patients who underwent allopurinol tolerance induction. Figures in the first three columns are mean +/- SD. 


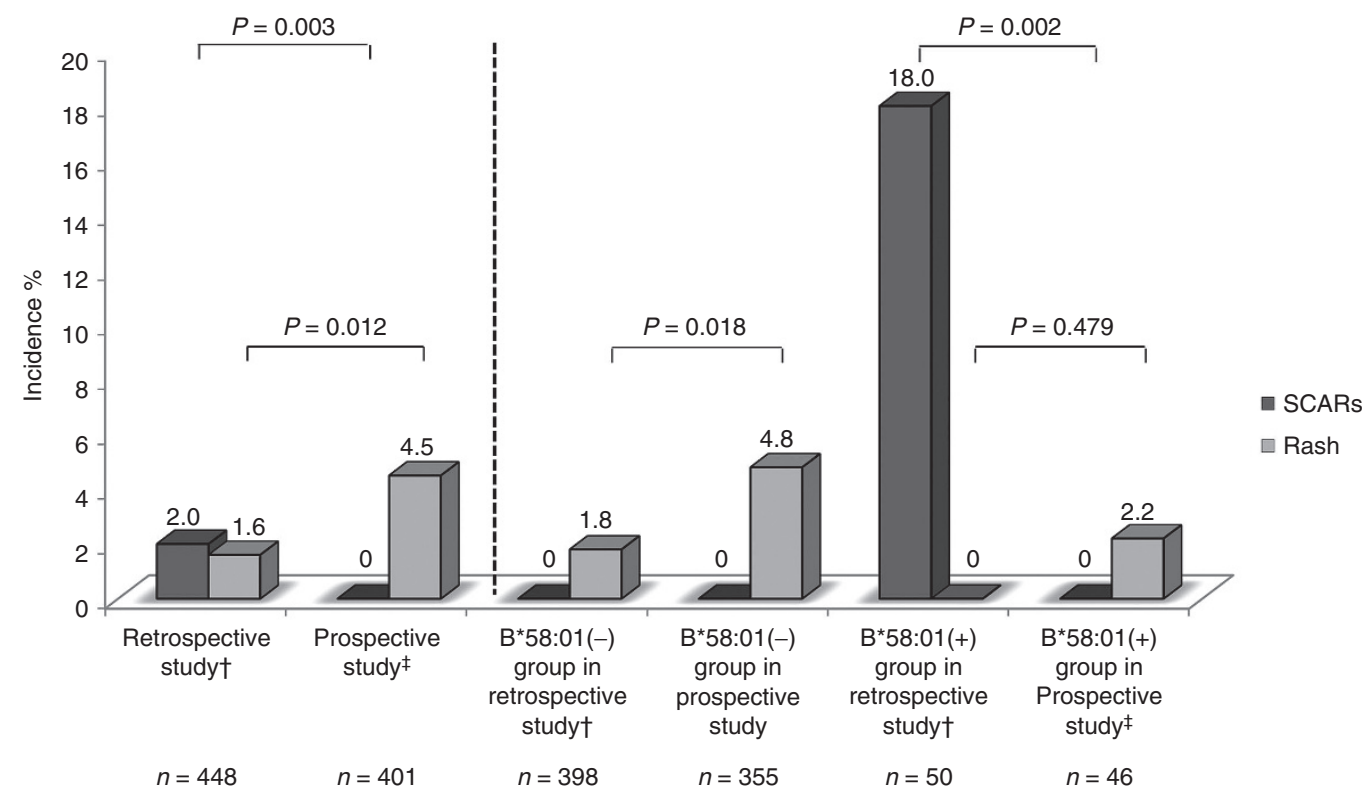

Figure 3 Comparison of the incidences of allopurinol hypersensitivity reactions with those in a previous retrospective study. Incidences of severe cutaneous adverse reaction (SCAR) were significantly reduced in the prospective study as compared with the previous retrospective study. ${ }^{+}$From ref. 15 . ${ }^{\ddagger}$ Treated with a tolerance induction protocol or alternative medications. ${ }^{20}$

differences in sex, age, underlying disease, laboratory test results, or dose of administered allopurinol between the 17 patients with hypersensitivity reaction and the 338 patients without reaction.

\section{Comparison of incidence of allopurinol hypersensitivity reactions with that of a previous retrospective study}

To test the preventive effect of HLA-B`58:01 screening for allopurinol hypersensitivity reaction, we compared the incidence of allopurinol hypersensitivity in the current prospective study with that from the HLA- ${ }^{\star} 58$ :01 screening in our previous retrospective cohort study. ${ }^{15}$ Whereas the previous retrospective study revealed a $2.0 \%$ incidence of allopurinol-induced SCARs, no SCARs developed after 90 days of allopurinol administration after the introduction of HLA-B ${ }^{\star} 58$ :01 screening and a tolerance induction protocol (2.0 vs. $0 \% ; P=0.003$; Figure 3 ). Of the 46 HLA-B ${ }^{\star} 58: 01$-positive patients, 8.28 patients were expected to have SCARs, considering the $18 \%$ incidence of SCARs in the previous retrospective cohort study of a similar population. However, none of the HLA-B ${ }^{\star} 58$ :01-positive 46 patients had SCARs. Compared with the previous study, this demonstrates a significant reduction of SCAR incidence in the 46 HLA-B ${ }^{\star} 58: 01$-positive patients with the help of HLA-B screening (18 vs. $0 \% ; P=0.002$; Figure 3 ).

By contrast, compared with the previous retrospective cohort study, a higher incidence of maculopapular rash was observed in the current prospective study, both in total study subjects and in patients without HLA-B ${ }^{\star} 58: 01$ (1.6 vs. $4.5 \% ; P=0.012$ and 1.8 vs. $4.8 \% ; P=0.018$, respectively; Figure 3 ).

\section{Subgroup analysis of patients treated with allopurinol}

Among 385 patients treated with allopurinol, 30 HLA-

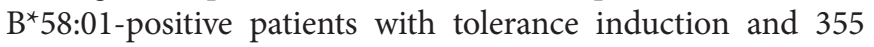

HLA-B ${ }^{\star}$ 58:01-negative patients were compared. There was no difference in age, sex, renal function, final allopurinol dose, or underlying diseases, including allergic disease, between the two subgroups (Table 1). Compared with the previous retrospective cohort study, ${ }^{15}$ a significant reduction of SCAR incidence was observed in the 30 HLA-B ${ }^{\star 58}$ :01-positive patients who underwent the allopurinol tolerance induction protocol (18 vs. $0 \% ; P=0.011$ ), in spite of using a similar final allopurinol dose $(115.00 \pm 56.24$ vs. $103.45 \pm 42.11 \mathrm{mg} ; P=0.304)$.

\section{DISCUSSION}

This study highlights the clinical usefulness of the HLA-B ${ }^{\star} 58: 01$ test in patients with CRI to prevent allopurinol-induced SCARs and the safety of allopurinol administration in patients without HLA-B ${ }^{\star} 58: 01$. Along with these findings, we also showed the potential benefit of a tolerance induction protocol, a gradual increase in allopurinol based on a slow oral desensitization protocol, as a preventive measure for patients at high risk for allopurinol-induced SCARs.

As a result of vigorous new drug developments in the recent era, morbidity and mortality due to adverse drug reactions have become a huge burden. ${ }^{24}$ Drug allergy is considered immunologically mediated and classified into categories I-IV by the Gell and Coombs classification. ${ }^{25}$ Delayed drug hypersensitivity reaction is mainly related to type IV hypersensitivity, that is, a T-cell-mediated reaction. ${ }^{25} \mathrm{SJS} / \mathrm{TEN}$ is especially related to CD8+ T lymphocytes and the associated class I HLA-restricted processes such as HLA-A, $-\mathrm{B}$, and $-\mathrm{C}^{26}$

Many studies have been performed to demonstrate the association between certain drugs and HLA alleles in the development of SCARs, and a strong correlation was reported for several drugs. ${ }^{9,11,13-15,27,28}$ Therefore, many researchers recently 
have attempted to prevent the occurrence of SCARs through HLA screening, despite the long-lasting belief that a type B reaction is unpredictable. ${ }^{27,29}$ As an example of this attempt, Chen et al. ${ }^{27}$ completely prevented the development of SJS and TEN by screening for HLA-B ${ }^{\star} 15: 02$ before carbamazepine use in 4,877 southeast Asian subjects, which proved the significant usefulness of HLA-B ${ }^{\star} 15: 02$ screening. In the PREDICT-1 study, HLA-B`57:01, a known risk marker for abacavir hypersensitivity reaction, was screened for in 1,956 human immunodeficiency virus (HIV)-infected patients before abacavir administration. With this screening test to exclude the highrisk patients from abacavir medication, abacavir hypersensitivity reaction was significantly reduced $(7.8$ vs. $3.4 \% ; P<0.001)$, and an immunologically confirmed hypersensitivity reaction never occurred in the prospective screening group. ${ }^{29}$ This finding suggests the clinical usefulness of giving the HLA screening test before abacavir administration in HIV patients.

The current study revealed the usefulness of the HLA$B \star 58: 01$ screening test before allopurinol use for the prevention of allopurinol-induced hypersensitivity responses in subjects with renal insufficiency, which is consistent with the finding of our previous retrospective cohort study, performed in a similar population at the same institution. ${ }^{14,15}$

Most exciting is that our study is the first to provide substantial evidence to support the effectiveness of a tolerance induction protocol to prevent the development of allopurinol-induced hypersensitivity response in a group of high-risk patients. This unique design is based on the idea that primary prevention of hypersensitivity reaction can be attained by inducing immunological tolerance with the introduction of a "desensitization protocol." The desensitization method is used to induce tolerance artificially in subjects with a drug allergy in order to prevent recurrence. In clinical fields, a rapid desensitization method is widely used for Immunoglobulin E-mediated hypersensitivity reactions by drugs such as $\beta$-lactam antibiotics or antineoplastic drugs, with a high success rate. ${ }^{16}$ Rapid desensitization induces temporary unresponsiveness to a drug antigen by administering the drug from an extremely low concentration to the usual concentration by doubling the dose in 15-min intervals for several hours. ${ }^{16} \mathrm{~T}$-cell-mediated hypersensitivity is, however, a major mechanism in delayed hypersensitivity reaction, which requires dozens of days to increase the dose step by step for tolerance induction. ${ }^{30}$ In the current study we used a 28-day slow tolerance induction protocol adapted from the desensitization protocol described by Fam et al., ${ }^{21}$ which showed successful tolerance induction in $78 \%$ of hyperuricemic patients with allopurinol-induced maculopapular eruption, and borrowed the idea from the fact that allopurinol administration by a slow desensitization protocol could avoid activating already existing memory cells into effector cells. We speculated that slow, gradual escalation of the drug based on a tolerance induction protocol might help to skip the generation of effector and memory $\mathrm{T}$ cells, although the existence of those cells was not verified in the study. A previous study reported that the starting dose was a risk factor for allopurinol hypersensitivity syndrome. ${ }^{31}$ In addition, Yun et al. ${ }^{32}$ reported that a high concentration of allopurinol is an important factor for the generation of drug-specific $\mathrm{T}$ cells in the presence of the HLA-B ${ }^{\star 58: 01}$ allele. Based on our previous retrospective cohort study, the current study showed that potentially 5.4 new cases of SCAR among $30 \mathrm{HLA}^{\star}{ }^{\star} 58: 01$ positive patients were successfully prevented with the introduction of the tolerance induction protocol. This finding suggests the promising role of the tolerance induction protocol as a preventive measure for delayed hypersensitivity for a group of high-risk patients. The mechanistic details of T-cell regulation by slow gradual antigen administration in the desensitization method used in patients with drug allergy still remain unclear, however, and the mechanism of achieving tolerance by slow, gradual administration in high-risk but allopurinol-naive subjects could be different from that in patients who are already sensitized.

Benzbromarone and febuxostat are alternatives to allopurinol. Benzbromarone has a urate-lowering effect by inducing renal uric acid leakage and can be used in patients with mild to moderate renal insufficiency, but it may be hepatotoxic. ${ }^{33}$ Febuxostat has emerged as a new nonpurine selective inhibitor of xanthine oxidase that is expected to be useful in patients with mildly decreased renal function..$^{34}$ In this study benzbromarone was administered in 11 patients and febuxostat in 5 patients with the HLA-B ${ }^{\star 58: 01}$ allele. During the 90 -day period of drug administration, none of them developed drug hypersensitivity reactions, including SCARs. This indicates that benzbromarone or febuxostat may be used as an alternative drug to allopurinol in a group of high-risk patients with HLA- $B^{\star} 58: 01$.

From 2005 to 2010, the mean medical cost per person with an allopurinol-induced SCAR was US $\$ 48,255$ in the same hospital where this study was performed (data not shown). Considering the incidence of SCAR in our previous retrospective cohort study, ${ }^{15}$ about US $\$ 400,000$ would have been spent for 8.28 patients with potential SCARs among 401 study subjects without HLA-B ${ }^{\star} 58$ :01 screening. Therefore, the cost of the HLA-B allele test for 401 study subjects (US $\$ 19,667$ ) was only $4.9 \%$ of the estimated cost of treating potential SCARs.

In this study one patient in the HLA-B ${ }^{\star} 58: 01$-positive group developed allopurinol-induced maculopapular eruption, even with application of the drug tolerance induction protocol. However, this patient had very mild rash without mucosal involvement or any other symptoms suggesting SCAR, and the skin lesions rapidly resolved in 4 days after ceasing the administration of allopurinol. Considering that SCARs usually progress to full-blown features even after discontinuation of the causative agents, it is less likely that the case might have been a potential SCAR.

In contrast to SCARs, the incidence of maculopapular eruption in this study population increased as compared with that of the previous retrospective cohort study (Figure 3). It is very likely, however, that the incidence of mild hypersensitivity might have been underestimated in the previous 
retrospective cohort study, considering the nature of a retrospective study.

There are some limitations in this study. The study proved statistical significance, but its sample size was not large enough to generalize the study result to the general population. In spite of this limitation, the relatively high frequency of HLA-B ${ }^{\star} 58: 01$ in the Korean population (12.2\%) enabled us to attain clinical significance with a relatively small number of subjects. Another limitation is the lack of information on data about the incidence of allopurinol-induced SCARs in other populations with or without CRI and the lack of a concurrent control group of HLA-B ${ }^{\star} 58$ :01-positive subjects starting with the usual dose of allopurinol, for ethical reasons. Instead, a previous retrospective cohort study done at the same institution was used as a historical control. The other limitation is that the final uric acid concentrations at 90 days were not vigorously controlled below $6 \mathrm{mg} / \mathrm{dl}$ because uric acid concentration for the treatment of hyperuricemia in chronic kidney disease is still controversial, especially when the patient is treated with allopurinol. Therefore, a largescale replication study in another population would be needed to further support the clinical significance of HLA-B ${ }^{\star} 58: 01$ screening and the use of an intolerance induction protocol for the primary prevention of SCARs in high-risk patients.

In conclusion, the current study demonstrated the usefulness of the HLA-B ${ }^{\star}$ 58:01 screening test before allopurinol use for the prevention of allopurinol-induced SCARs in subjects with renal insufficiency, which is consistent with the finding of our previous retrospective cohort study, performed in a similar population at the same institution. Our studies also highlight that tolerance induction in a group of high-risk patients could be an effective strategy to significantly reduce the morbidity and mortality related to SCARs.

\section{SUPPLEMENTARY MATERIAL}

Supplementary material is linked to the online version of the paper at http://www.nature.com/gim

\section{ACKNOWLEDGMENTS}

This study was supported by a grant from the Korea Health 21 R\&D Project, Ministry of Health \& Welfare, Korea (grant 03-PJ10-PG13GD01-0002). We appreciate statistical consultation provided by the Medical Research Collaborating Center at the Seoul National University College of Medicine / the Seoul National University Hospital. Medical Research Collaborating Center at Seoul National University College of Medicine \& Seoul National University Hospital provided the sample size calculation in the design of this study.

\section{DISCLOSURE}

The authors declare no conflict of interest.

\section{REFERENCES}

1. Roujeau JC, Stern RS. Severe adverse cutaneous reactions to drugs. N Engl J Med 1994;331:1272-1285

2. Finkelstein $Y$, Macdonald EM, Li P, Hutson JR, Juurlink DN. Recurrence and mortality following severe cutaneous adverse reactions. JAMA 2014;311: 2231-2232.
3. Mockenhaupt M, Viboud C, Dunant A, et al. Stevens-Johnson syndrome and toxic epidermal necrolysis: assessment of medication risks with emphasis on recently marketed drugs. The EuroSCAR-study. J Invest Dermatol 2008;128:35-44.

4. Wortmann RL. Gout and hyperuricemia. Curr Opin Rheumatol 2002;14: 281-286.

5. Pluim HJ, van Deuren M, Wetzels JF. The allopurinol hypersensitivity syndrome Neth J Med 1998;52:107-110.

6. Hung $\mathrm{SI}$, Chung WH, Liou LB, et al. HLA-B*5801 allele as a genetic marker for severe cutaneous adverse reactions caused by allopurinol. Proc Natl Acad Sci USA 2005;102:4134-4139.

7. Auböck J, Fritsch P. Asymptomatic hyperuricaemia and allopurinol induced toxic epidermal necrolysis. Br Med J (Clin Res Ed) 1985;290:1969-1970.

8. Singer JZ, Wallace SL. The allopurinol hypersensitivity syndrome. Unnecessary morbidity and mortality. Arthritis Rheum 1986;29:82-87.

9. Chung WH, Hung SI, Hong HS, et al. Medical genetics: a marker for StevensJohnson syndrome. Nature 2004;428:486.

10. Amstutz $U$, Shear NH, Rieder MJ, et al.; CPNDS clinical recommendation group. Recommendations for HLA-B*15:02 and HLA-A*31:01 genetic testing to reduce the risk of carbamazepine-induced hypersensitivity reactions. Epilepsia 2014;55:496-506.

11. Mallal S, Nolan D, Witt C, et al. Association between presence of HLA-B*5701, HLA-DR7, and HLA-DQ3 and hypersensitivity to HIV-1 reverse-transcriptase inhibitor abacavir. Lancet 2002;359:727-732.

12. Guo Y, Shi L, Hong H, Su Z, Fuscoe J, Ning B. Studies on abacavir-induced hypersensitivity reaction: a successful example of translation of pharmacogenetics to personalized medicine. Sci China Life Sci 2013;56:119-124.

13. Tassaneeyakul $W$, Jantararoungtong $T$, Chen $P$, et al. Strong association between HLA-B *5801 and allopurinol-induced Stevens-Johnson syndrome and toxic epidermal necrolysis in a Thai population. Pharmacogenet Genomics 2009;19:704-709.

14. Kang HR, Jee YK, Kim YS, et al.; Adverse Drug Reaction Research Group in Korea. Positive and negative associations of HLA class I alleles with allopurinolinduced SCARs in Koreans. Pharmacogenet Genomics 2011;21:303-307.

15. Jung JW, Song WJ, Kim YS, et al. HLA-B58 can help the clinical decision on starting allopurinol in patients with chronic renal insufficiency. Nephrol Dial Transplant 2011;26:3567-3572.

16. Castells M. Rapid desensitization for hypersensitivity reactions to chemotherapy agents. Curr Opin Allergy Clin Immunol 2006;6:271-277.

17. Miller SA, Dykes DD, Polesky HF. A simple salting out procedure for extracting DNA from human nucleated cells. Nucleic Acids Res 1988;16:1215.

18. Prasad VK, Yang SY. Allele assignment for HLA-A, -B, and -C genes to the Tenth International Histocompatibility Workshop cell lines. Tissue Antigens 1996;47:538-546.

19. Lee KW, Oh DH, Lee C, Yang SY. Allelic and haplotypic diversity of HLA-A, $-B,-C,-D R B 1$, and -DQB1 genes in the Korean population. Tissue Antigens 2005;65:437-447.

20. Hande KR, Noone RM, Stone WJ. Severe allopurinol toxicity. Description and guidelines for prevention in patients with renal insufficiency. Am J Med 1984;76:47-56.

21. Fam AG, Dunne SM, lazzetta J, Paton TW. Efficacy and safety of desensitization to allopurinol following cutaneous reactions. Arthritis Rheum 2001;44: 231-238.

22. Roujeau JC. The spectrum of Stevens-Johnson syndrome and toxic epidermal necrolysis: a clinical classification. J Invest Dermatol 1994;102:28S-30S.

23. Meyboom RH, Hekster YA, Egberts AC, Gribnau FW, Edwards IR. Causal or casual? The role of causality assessment in pharmacovigilance. Drug Saf 1997; 17:374-389.

24. Edwards IR, Aronson JK. Adverse drug reactions: definitions, diagnosis, and management. Lancet 2000;356:1255-1259.

25. Gomes ER, Demoly P. Epidemiology of hypersensitivity drug reactions. Curr Opin Allergy Clin Immuno/ 2005;5:309-316.

26. Nassif A, Bensussan A, Dorothée $G$, et al. Drug specific cytotoxic T-cells in the skin lesions of a patient with toxic epidermal necrolysis. J Invest Dermatol 2002;118:728-733.

27. Chen P, Lin JJ, Lu CS, et al.; Taiwan SJS Consortium. Carbamazepineinduced toxic effects and HLA-B*1502 screening in Taiwan. N Engl J Med 2011;364:1126-1133.

28. Alfirevic A, Jorgensen AL, Williamson PR, Chadwick DW, Park BK, Pirmohamed M. HLA-B locus in Caucasian patients with carbamazepine hypersensitivity. Pharmacogenomics 2006;7:813-818.

29. Mallal S, Phillips E, Carosi G, et al.; PREDICT-1 Study Team. HLA-B*5701 screening for hypersensitivity to abacavir. N Engl J Med 2008;358:568-579. 


\section{ORIGINAL RESEARCH ARTICLE}

30. Fam AG. Difficult gout and new approaches for control of hyperuricemia in the allopurinol-allergic patient. Curr Rheumatol Rep 2001;3:29-35.

31. Stamp LK, Taylor WJ, Jones PB, et al. Starting dose is a risk factor for allopurinol hypersensitivity syndrome: a proposed safe starting dose of allopurinol. Arthritis Rheum 2012;64:2529-2536.

32. Yun J, Mattsson J, Schnyder K, et al. Allopurinol hypersensitivity is primarily mediated by dose-dependent oxypurinol-specific T cell response. Clin Exp Allergy 2013;43:1246-1255.
JUNG et al | Screening test for HLA-B*58:01 and prevention of allopurinol-induced SCARs

33. Zhang $W$, Doherty $M$, Bardin $T$, et al.; EULAR Standing Committee for International Clinical Studies Including Therapeutics. EULAR evidence based recommendations for gout. Part II: management. Report of a task force of the EULAR Standing Committee for International Clinical Studies Including Therapeutics (ESCISIT). Ann Rheum Dis 2006:65:1312-1324

34. Becker MA, Schumacher HR Jr, Wortmann RL, et al. Febuxostat compared with allopurinol in patients with hyperuricemia and gout. N Engl J Med 2005:353:2450-2461. 